\title{
Signal Denoise Method Based on the Higher Order Cumulant and Local
}

\section{Tangent Space Mean Reconstruction}

\author{
Guangbin Wang ${ }^{1, a}$, Xuejun $\mathrm{Li}^{2, b}$, Ke Wang ${ }^{3, c}$ \\ ${ }^{1}$ Engineering Research Center of Advanced Mine Equipment, Ministry of Education, \\ Hunan University of Science and Technology, china \\ ${ }^{2}$ Hunan Provincial Key Laboratory of Health Maintenance for Mechanical Equipment, Hunan \\ University of Science and Technology,china \\ a jxxwgb@126.com bhnkjdxlxj@163.com 'wangkekeer@126.com
}

Keywords: Signal denoise; Higher order cumulant;Intrinsic dimension;Manifold learning;

\begin{abstract}
In signal denoise method to nonlinear time series based on principle manifold learning, reduction target dimension is chosen at random, which cause low efficiency. Local low dimensional manifold is obtained by the eigenvalue decomposition to the covariance matrix, but covariance belongs to the second order statistics and cannot reflect the nonlinear essential structure of signal, these reduce denoise efficiency and effect. In order to solve these problem, a new denoise algorithm based on the higher order cumulant and local tangent space mean reconstruction is proposed in this reserch. First, the signal's intrinsic dimension is obtained as dimension of reduction targets by maximum likelihood estimation. And then making use of restraining character to colored noise of high order cumulan,covariance matrix is constructed by high order cumulant function instead of second order moment function. The data outside intrinsic dimension space will be regarded as noise signal to be eliminated. Finanly the process of global array by affine transformation will be replaced by mean reconstruction, the data after denoise may be obtained in the inverse process of the phase space reconstruction. The effectiveness of the algorithm is verified through the denoise experiment in fan vibration signal with noise.
\end{abstract}

\section{Introduction}

When the mechanical system runs, the status signal with noise and interference are generally nonlinear and nonstationary, signal and noise have similar wide spectral characteristic.Traditional denoise methods are mainly filter technology based on linear smoothing, which can not produce very good noise reduction effect, so the design of nonlinear denoise filter becomes necessary. Phase space reconstruction is an important tool for nonlinear dynamic analysis. The denoise algorithm based on phase space reconstruction have singular spectrum method[1], local projective method[2-3] and so on,but they are iterative local neighborhood methods, not considering the global structure information of manifold.In 2000, Roweis and Seung had simultaneously published research papers about manifold learning in Science [4-5], proposed Isometric Feature Mapping (ISOMAP) [4] and Local Linear Embedding (LLE) [5] algorithms, and successfully applied them to graph recognition.As a starting point, researchers had launched a variety of algorithms,such as Laplace Mapping (LE) [6], Local Tangent Space Alignment ( LTSA) [7] and other algorithms[8]. To signal noise reduction, Yang Jianhong has proposed noise reduction algorithm based on phase reconstruction and manifold recognition[9] which also called LTSA denoise algorithm.

The LTSA denoise algorithm has better effcet by taking the appropriate parameters, but the choice of target dimension is stochastic and uncertain scope, local tangent space coordinates are obtained from singular value decomposition to neighbors second order statistic reflecting the linear correlation in the phase space, data may be distorted in the prosess of global array and signal reverse solution, these will lead to low efficiency and effect of noise reduction. In order to solve 
these question, signal denoise method based on the higher order cumulant and local tangent space mean reconstruction (The simple form is HOC-LTSMR) is proposed. First, the signal's intrinsic dimension is obtained as dimension of reduction targets by maximum likelihood estimation. And then making use of restraining character to colored noise of high order cumulan,covariance matrix is constructed by high order cumulant function instead of second order moment function. The data outside intrinsic dimension space will be regarded as noise signal to be eliminated. Finanly the process of global array by affine transformation will be replaced by mean reconstruction,the data after denoise may be obtained in the inverse process of the phase space reconstruction. Experiments on blower's vibrating signal show that this method has better denoise effect.

\section{Construction of the covariance matrix based on third order cumulant}

As one kind of nonlinear signal processing tool, higher order statistic can reflect the nonlinear structure of signal and system, and also better suppress the disturbance of noise. The higher order statistics's main application in the signal processing domain are as followed: the recognition of signal nonlinear characteristics, the reduction of colored Gaussian noise, the extraction of information deviation from the gaussian distribution, the reconstruction of non-minimum phase signal and so on [10-12]. The definition of higher order moment and higher orde cumulant to random variables are as followed.

Suppose $\{x(n)\}$ is the zero average value and $k$ order stationary random process, the $k$ order moment is defined as

$$
m_{k}^{x}\left(\tau_{1}, \tau_{2} \cdots \tau_{k-1}\right)=E\left\{x(n) x\left(n+\tau_{1}\right) \cdots x\left(n+\tau_{k-1}\right)\right\}
$$

$E\{\bullet\}$ expresses the operation operator striving for the mathematic expectation.According to higher order moment and higher order cumulant transformation relation, the higher order cumulant of $\{x(n)\}$ expresses as follows

$$
C_{k}^{x}\left(\tau_{1}, \tau_{2}, \cdots \tau_{k-1}\right)=m_{k}^{x}\left(\tau_{1}, \tau_{2}, \cdots \tau_{k-1}\right)-m_{k}^{G}\left(\tau_{1}, \tau_{2}, \cdots \tau_{k-1}\right)
$$

Where $m_{k}^{G}\left(\tau_{1}, \tau_{2}, \cdots \tau_{k-1}\right)$ is the $k$ order moment function of the gauss signal having the same mean and autocorrelation function as $\{x(n)\}$.when $\{x(n)\}$ is the gauss signal and $k \geq 3, m_{k}^{x}\left(\tau_{1}, \tau_{2}, \cdots \tau_{k-1}\right)=m_{k}^{G}\left(\tau_{1}, \tau_{2}, \cdots \tau_{k-1}\right), \quad C_{k}^{x}\left(\tau_{1}, \tau_{2}, \cdots \tau_{k-1}\right)=0$. The first order cumulant expresses signal's mean, the second order cumulant expresses signal's autocorrelation function. The third order cumulant's expression is as follows:

$C_{3}^{x}\left(\tau_{1}, \tau_{2}\right)=m_{3}^{x}\left(\tau_{1}, \tau_{2}\right)-m_{1}^{x}\left[m_{2}^{x}\left(\tau_{1}\right)+m_{2}^{x}\left(\tau_{2}\right)+m_{2}^{x}\left(\tau_{1}-\tau_{2}\right)\right]+2\left(m_{1}^{x}\right)^{3}$

In the above equation, $k$ order moment's estimate expression is as followed

$$
m_{1}^{x}(\tau)=\frac{1}{N} \sum_{n=1}^{N} x(n), m_{2}^{x}(\tau)=\frac{1}{N} \sum_{n=1}^{N} x(n) x(n+\tau), m_{3}^{x}\left(\tau_{1}, \tau_{2}\right)=\frac{1}{N} \sum_{n=1}^{N} x(n) x\left(n+\tau_{1}\right) x\left(n+\tau_{2}\right)
$$

The covariance matrix $A$ is constructed by $(A)_{i, j}=C_{3}^{x}(i, j)$.

\section{Signal denoise method based on HOC-LTSMR}

In the singular spectrum denoise theory, by the singular value decomposition, the subspaces composed of the eigenvectors corresponding to the different eigenvalue form the ellipsoid or superellipsoid, eigenvalue is equal to the square of the ellipsoid half axle's square and the eigenvector has assigned half axle's direction. In the phase space,the The optimal projection direction is decided by eigenvectors corresponding to maximum eigenvalues. If the eigenvalue is small, the corresponding eigenvectors can be considered as noise to eliminate. Here the $d$ biggest right singular vectors of the centralized neighborhood matrix form the local manifold of the phase space and carry the system's important information,other eigenvectors corresponding to the smaller eigenvalues are considered to generate from the noise and the disturbance, and should be assigned zero. 
In this paper, the covariance matrix is constructed with the third order cumulant instead of second order moment function, intrinsic dimension by the maximum likelihood estimation inrcreases the efficiency of the dimension reduction, global mean reconstruction reduce the effects of the interference, which has realized denoise algorithm's optimization. Given time series signal $S=\left[s_{1}, s_{2}, \cdots s_{n}\right]$, The steps of signal denoise method based on HOC-LTSMR follows,

1). The best embedding dimension $m$ and delay time $\tau$ are obtained by $\mathrm{C}-\mathrm{C}$ algorithm, phase matrix after the reconstruction is $P \in R^{m \times[n-(m-1) t]}$, it's element is

$P_{f, k}=s_{k+(f-1) t}$

where, $f \in[1, m], k \in[1, n-(m-1) t]$. The intrinsic dimension $d$ is obtained by MLE, and is also regarded as reduction target dimension.

2). Select $k$, construct the neighborhood matrix $P_{f}^{k}$ of each point $P_{f}$.

3). Calculate the third order cumulant $C_{3}^{p}$ to the neighborhood matrix $P_{f}^{k}$, obtain the covariance matrix $\left(A_{f}^{k}\right)_{i, j}=C_{3}^{x}(i, j)$.

3). Make $A_{f}^{k}$ orthogonal singular value decomposition,thus $A_{f}^{k}=S \Sigma C^{T} . \quad S$ and $C$ are respectively left and right singular vector matrix of covariance matrix $A_{f}^{k}\left(A_{f}^{k^{T}}\right), \Sigma$ is $n \times n$ diagonal matrix, $\Sigma=\operatorname{diag}\left(\sigma_{1}, \sigma_{2}, \cdots \sigma_{k}\right), \sigma$ is singular value. Make the appropriate changes to $A_{f}^{k}=S \Sigma C^{T}$,obtain $A_{f}^{k} C=S \Sigma . A_{f}^{k} C$ is the track matrix by orthogonal projection, projection space $C$ composed of $\left\{C_{i}\right\}$ is local tangent space.

4). Repeat step 3, we can obtain $N_{p}=n-(m-1) \tau$ neighborhood matrices corresponded to $P_{j}$ after denoise.

5). For each phase point $P_{j}$, at first we find vectors $y_{i}^{p}$ after denoise from $N_{p}$ matrices, then obtain the global vector coordinates $y_{i}$ by averaging each phase point $P_{j}$, at last obtain phase martric $Y$ after denoise.

6). Reverse calculate one-dimensional signal by Eq.6.

$\hat{x}_{i}=\frac{\sum_{t \in\left\{I_{i}(j, k)\right\}} Y_{t}}{C_{i}} \quad i=1,2, \cdots, n$

Where, $\quad\left\{I_{i}(j, k)\right\}$ express all elements set satisfied $k+(j-1) \tau=i, j \in[1, m]$, $k \in\left[1, N-(m-1) \tau, \quad C_{i}\right.$ is number of elements in $\left\{I_{i}(j, k)\right\}$.

3. Study on denoise experiment

coupling bearing to the vibration signal of large fan

Reference 9 has proven that LTSA algorithm has good noise reduction effect to chaotic signals with white noise. In this experiment, vibration acceleration data with color noise is collected from cantilever fan in an aluminum company. Fan rated speed is $735 \mathrm{r} / \mathrm{min}$, motor power is $475 \mathrm{KW}$. Fan system is simplified as Fig.1, motor and bearing are fixed in cement concrete base, fan is fixed to the shaft, motor drives fan through shaft and coupling. Fan runs in big dust and electromagnetic interference environment, the vibration signals reflected equipment status contain much noise.In order to obtain effective signals, denoise must be 
firstly excuted.In Fig.2, the measuring point of sensor near the fan side in the vertical direction. In the power spectrum, SHG and THG are very strong (base frequency is $12.25 \mathrm{HZ}$ ), this shows that system has severe rub fault. The purpose of denoise is to highlight SHG and THG components and reduce the other frequency components.
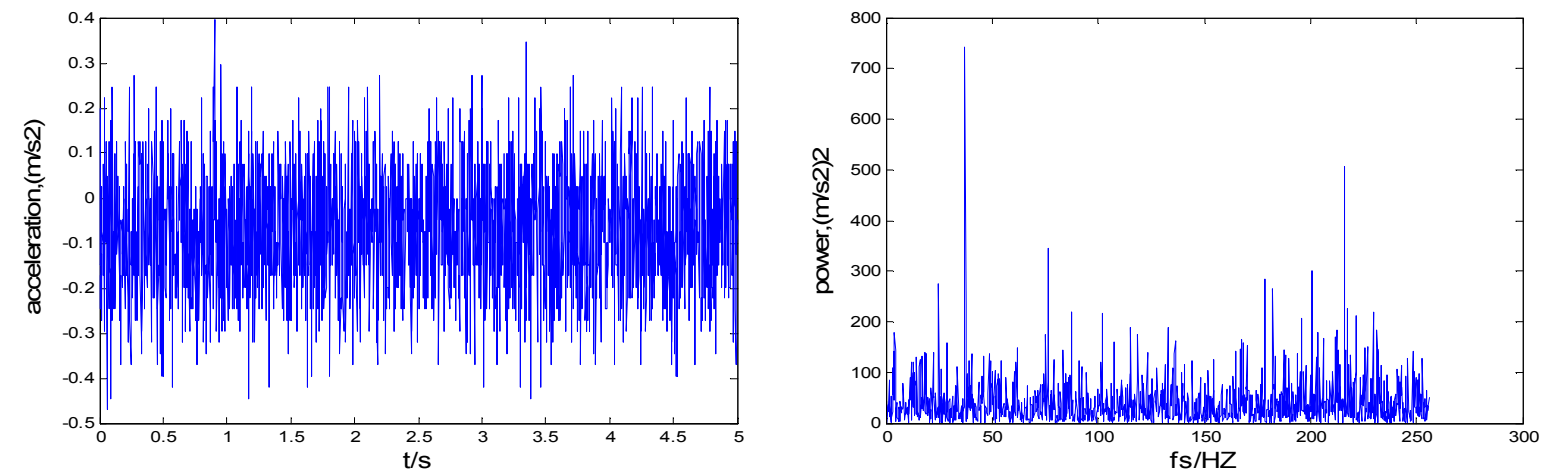

Fig.2 Time-domain waveform and power spectrum of fan fault signal

Fig.3 and Fig.4 are time-domain waveform and power spectrum of signal after denoise with LTSA and HOC-LTSMR algorithms $(k=20, d=6)$.Comparison with Fig.2, vibration amplitude of signal after denoise decrease, SHG and THG in power spectrum become more prominent. We can see from the figure, HOC-LTSMR method has better denoise effect.
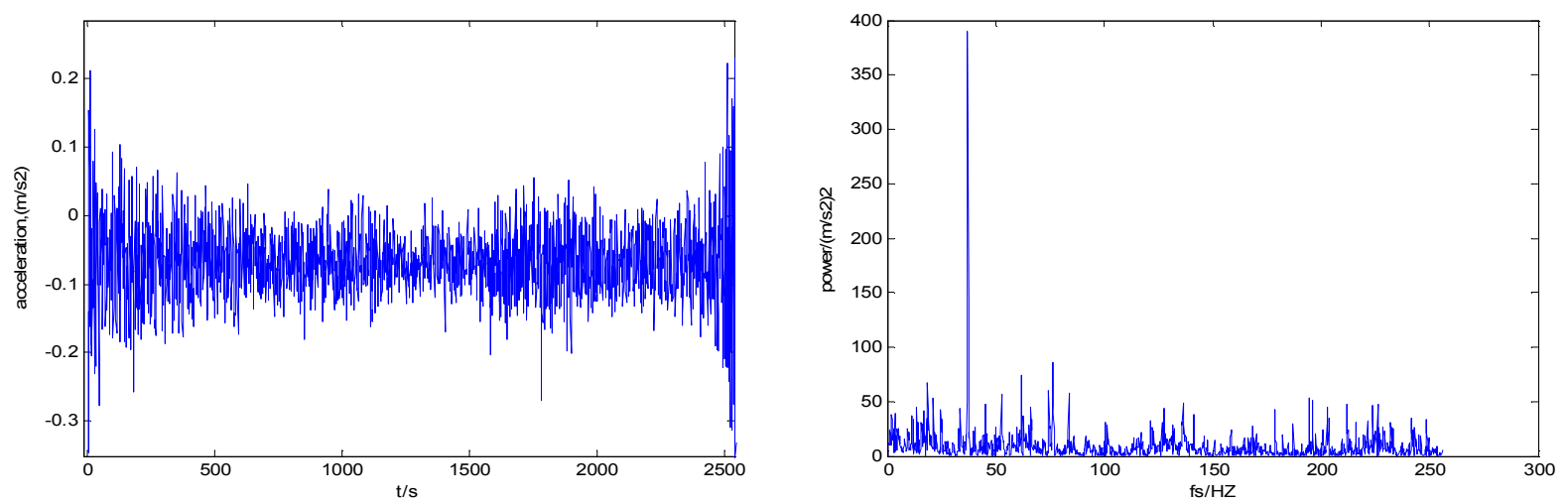

Fig.3 Time-domain waveform and power spectrum after noise reducation with LTSA
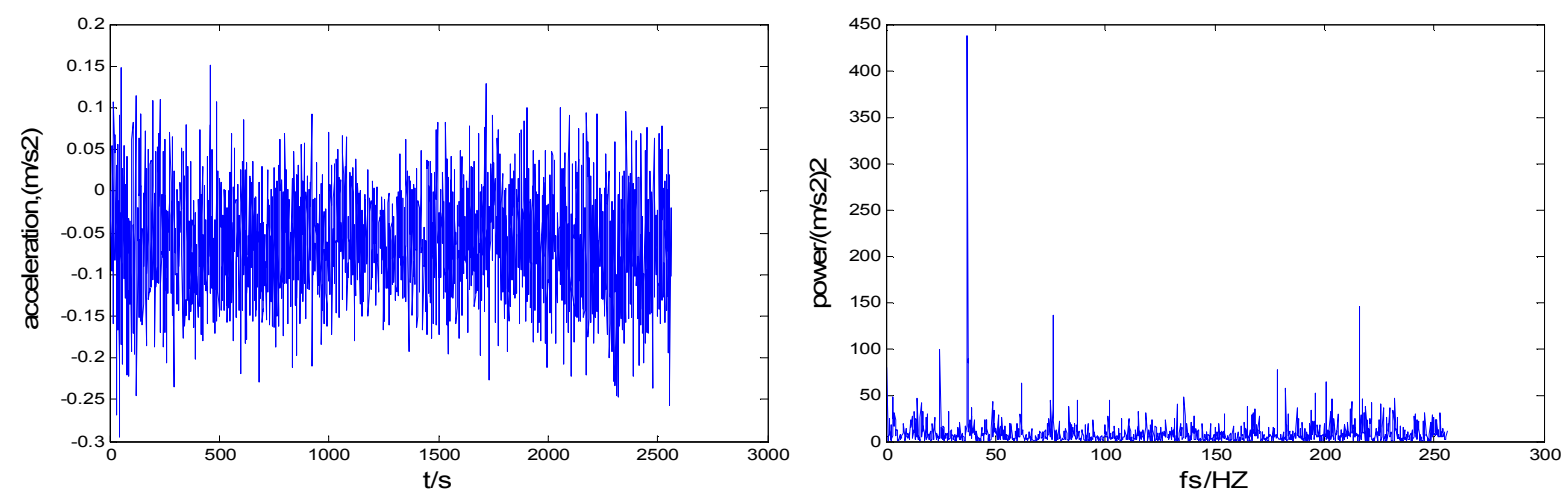

Fig.4 Time-domain waveform and power spectrum after noise reducation with HOC-LTSMR 


\section{Conclusion}

In the denoise algorithm based on LTSA, reduction target dimension are chosen at random, local tangent space coordinates are obtained from singular value decomposition to neighbors second order statistic reflecting the linear correlation in the phase space,and phase space data may be distorted in the prosess of dimension reducation, global array and signal reverse solution, they make efficiency and effect of noise reduction lower.we propose a new denoise algorithm named HOC-LTSMR, experiments for vibration signals with the colored noise show that this algorithm has better noise reduction effect than LTSA algorithm.

\section{Acknowledgment}

Financial support from Aid program for Science and Technology Innovative Research Team in Higher Educational Institutions of Hunan Province, the CEEUSRO special plan of Hunan province (2010XK6066),National Natural Science Foundation of China (51175170), The Industrial Cultivation Program of Scientific and Technological Achievements in Higher Educational Institutions of Hunan Province (10CY008), Natural Science Foundation of Hunan Province Key Project (09JJ8005), are gratefully acknowledged.

\section{References}

[1] K.Shin, J.K.Hammond,P.R.White:Mechanical Systems and Signal Proeessing, 13(1999),p.115 $-124$.

[2] $\mathrm{Xu}$ jinwu,Lu yong,Wang haifeng: Chinese Journal of Mechanical Engineering,,39(2003),p.1 46-150

[3] J.Sun, J.Zhang, M.Small:Signal Processing,88(2008),1881-1888

[4] J.B.Tememnaum,V.Silva, J.C.Langford: Science,290(2000),2319-2323.

[5] S.Roweis, L.Saul:Science,290(2000),2323-2326.

[6] M.Belkin, P.Niyogi: Neural Computation. 15(2003),1373-1396

[7] Zhang Zhenyue,Zha Hongyuan: SIAM Journal on Scientific Computing,26(2005),313-338

[8] He Xiaofei, P.Niyogi: Advances in Neural Information Processing Systems 16.MIT Press,ca mbrifge,MA,(2004)

[9] Yang jianhong,Xu jinwu,Yang debin,et al:Chinese Journal of Mechanical Engineering, $42(2$ 006):154-158

[10] Nikias C.L, Mendel J.M. IEEE Signal Processing Magazine, 1993, 10: 7-11.

[11] Heidari S, Nikias C.L. 1993 Conference Record of the Twenty-Seventh Asilomar Confere nce on Signals Systems and Computers Pacific Grove(CA,USA): IEEComputer Society Pre ss. 1993, 1: 466-470.

[12] Schittenkopf C, Deco G. Testing nonlinear Markovian hypotheses in dynamical systems. Physica D, 1997, 104: 61-74. 